\title{
Article
}

\section{The incubation periods, the critical immunisation threshold and some other predictors of SARS-CoV-2 disease for different location and different climate countries}

\author{
Marwan Al-Raeei \\ Faculty of Sciences, Damascus University, Damascus, Syrian Arab Republic; mhdm-ra@scs-net.org \\ Received: 21 March 2021; Accepted: 25 May 2021; Published: 29 June 2021.
}

\begin{abstract}
We estimate the incubation period values and other forecasting predictors of SARS-CoV-2 for different countries located in different geographical locations of the earth and each one has a certain climate. The considered countries are the United States, Russia, the United Kingdom, Brazil, Spain, Bahrain, Egypt, Iran, Cyprus, India, France, and the Syrian Arab Republic. For estimating of the forecasting predictors values, we use the SEIR epidemic model and Runge-Kutta simulation method. The estimations are done up to the beginning of 2021 in aforementioned countries based on the collected data in these countries. We find that the incubation period values of SARS-CoV-2 are located between 2.5 days which returns to Bahrain and 10 days which returns to some countries in middle east. Also, we find that the average value of this period is about 6.5 days for the different location countries. Besides, we find that the average values of SARS-CoV-2 critical immunisation threshold, SARS-CoV-2 basic reproduction number and SARS-CoV-2 steady state population are $0.5,2.3$ and 0.5 respectively.
\end{abstract}

Keywords: Incubation Period; Covid-19; Runge-Kutta method; SEIR model; Pandemic; Numerical simulation.

\section{Introduction}

$\mathbf{A}$ $t$ the end of 2019, a new class of SARS (Severe acute respiratory syndrome) virus disease caused by a new type of the corona viruses was discovered in Wuhan, China which named later SARS-CoV-2 (Severe acute respiratory syndrome-Corona Virus-2) or the new coronavirus disease and this disease spread over the world and caused more than three millions deaths over the world up to the writing of this article. Lots of studies discussed the spreading and forecasting of SARS-CoV-2 disease and influence of the disease on different locations. For instance, Lounis and Bagal [1] found the parameters of the SIR model for Algeria. Neto et al., [2] discussed the modelling of spreading of the disease for São Paulo in Brazil. Ebohon et al., [3] discussed the influence of SARS-CoV-2 on the education in Nigeria. Ganiny and Nisar [4] discussed the spreading of the disease in Indian regions. Aidoo et al., [5] discussed the modelling of SARS-CoV-2 incidence in the African sub regions using smooth transition autoregressive model. Other studies applied SIRD (susceptible-infected-recovered-dead) epidemic model for the studying the forecasting and spreading of SARS-CoV-2 disese [6-10] in addition to finding the indicators of the model for the disease. Also, in other studies, the SEIR (susceptible-exposed-infected-recovered) epidemic model was applied for the forecasting of SARS-CoV-2 disease [11-14] for different scenarios.

In general, the epidemiology forecasting model was suggested by Kermack [15] for the first time as a simple SIR epidemic model and other epidemiology compartmental forecasting models were derived based on this model such as SEIR model [16-22], SIRD model [9,10] and SVEIS (susceptible-vaccinated-exposed-infected- susceptible) model [23] which takes the vaccination into account. In this work, we use the SEIR model for finding some important predictors of SARS-CoV-2 by simulating the previous model using the numerical analysis methods. The first predicator which we focus on in this work is the period of incubation, which is one of the most important indicators of the spreading of a specific pandemic such as SARS-CoV-2, this period represents the average time of the incubation from the exposing and this period gives the ratio between the exposed population to the rate of the infectious population when we eliminate other infected reasons.

There are two methods for determining the values of the incubation period. The first one is observation of exposed persons by medical observer and the other method is theoretical method which estimates incubation 
period values based on one of compartmental models in epidemiology and collected data of total cases of a specific pandemic. The theoretical method is more preferable because it eliminates the contacts between patients and doctors or nurses. In this work, the period of incubation values of SARS-CoV-2 are estimated based on the numerical analysis methods for different location countries. The other considered predictors which we focus on in this study are the population steady state, which represents the ratio between the average age of infection and the average age at which every individual in the model is assumed to die, the critical immunisation threshold, which is the minimum of the proportion of the population that is immune, and the basic reproduction number, which represents the expected cases which is generated by one infectious case in a certain population with a specific disease. We calculate all of the previous indicators based on the same method via the SEIR model. The first two equations of the SEIR model are non-linear equations [24] and describe the change of the susceptible population and the change of the exposed population with respect to the time and the others equations describe the rate of the infections population and the recovery population in respect to the time. The four equations of the SEIR epidemiological model are given as follows:

$$
\begin{aligned}
& \frac{d S(t)}{d t}=-\frac{\xi_{2}}{N} I(t) S(t)+\xi_{1}[N-S(t)], \\
& \frac{d E(t)}{d t}=-\left(\xi_{1}+\xi_{3}\right) E(t)+\frac{\xi_{2}}{N} I(t)(t), \\
& \frac{d I(t)}{d t}=-\left(\xi_{1}+\xi_{4}\right) I(t)+\xi_{3} E(t), \\
& \frac{d R(t)}{d t}=-\xi_{1} R(t)+\xi_{4} I(t),
\end{aligned}
$$

where $N$ is the population number, $\xi_{1}, \xi_{2}, \xi_{3}$ and $\xi_{4}$ are parameters and $S(t), I(t), R(t)$ and $E(t)$ are the susceptible population of the pandemic individual, the infectious population of the pandemic individual, the recovered cases pandemic individual and the exposed population of the pandemic individual respectively. The previous populations are governed under the following conservation condition:

$$
\frac{d R(t)}{d t}+\frac{I(t)}{d t}+\frac{d E(t)}{d t}+\frac{d S(t)}{d t}=0 .
$$

In this study, we apply previous epidemiology model for estimating period of incubation, population steady state, critical immunization threshold and basic reproduction number of the new corona virus disease in the United States, where the first case of the pandemic was observed in January 2020, Russia, where the first case of the pandemic was observed in January 2020, the United Kingdom, where the first case of the pandemic was observed in January 2020, Brazil, where the first case of the pandemic was observed in February 2020, Spain, where the first case of the pandemic was observed in January 2020, Bahrain, where the first case of the pandemic was observed in February 2020, Egypt, where the first case of the pandemic was observed in February 2020, Iran, where the first case of the pandemic was observed in February 2020, Cyprus, where the first case of the pandemic was observed in March 2020 and the Syrian Arab Republic, where the first case of the pandemic was observed in March 2020. The aforementioned countries are considered located in different locations in all of the worldwide. In the Section 2, we illustrate the principle of the method used to find the predicators using the applied epidemiological model and in the Section 3, we illustrate the results and the discussion of the results while in the last section, we illustrate the conclusion of the results.

\section{Computational Implementations}

We collected the observed data of new corona virus disease recorded in the United States, Russia, the United Kingdom, Brazil, Spain, Bahrain, Egypt, Iran, Cyprus, France, India and the Syrian Arab Republic up to the binging of 2021. After that, We applied Runge-Kutta (R-K) method on the SIER epidemiologic model where in the light of this numerical method, the compartmental populations individual are written as follows [25]:

$$
\begin{aligned}
& S\left(t_{n+1}\right) \approx S\left(t_{n}\right)+\tau \sum_{m-1}^{m-z} l^{1} m k^{1} m, \\
& I\left(t_{n+1}\right) \approx I\left(t_{n}\right)+\tau \sum_{m-1}^{m-z} l^{2} m k^{2} m,
\end{aligned}
$$




$$
\begin{aligned}
& R\left(t_{n+1}\right) \approx R\left(t_{n}\right)+\tau \sum_{m-1}^{m-z} l^{3} m k^{3} m, \\
& E\left(t_{n+1}\right) \approx E\left(t_{n}\right)+\tau \sum_{m-1}^{m-z} l^{4} m k^{4} m,
\end{aligned}
$$

with the following R-K functions:

$$
\begin{aligned}
& k_{1}^{1}=f_{1}\left(t_{n}, S_{n}\right), \\
& k_{1}^{1}=f_{1}\left(t_{n}+c_{2}^{1} \tau, S_{n}+\tau\left(a_{21}^{1} k_{1}^{1}\right)\right) \\
& k_{1}^{z}=f_{1}\left(t_{n}+c_{z}^{1} \tau, S_{n}+\tau\left(a_{z 1}^{1} k_{1}^{1}+a_{z 2}^{1} k_{2}^{1}+a_{z 3}^{1} k_{3}^{1}+\ldots+a_{z, z-1}^{1} k_{z-1}^{1}\right),\right. \\
& k_{1}^{2}=f_{2}\left(t_{n}, I_{n}\right) \\
& k_{2}^{2}=f_{2}\left(t_{n}+c_{2}^{2} \tau, I_{n}+\tau\left(a_{21}^{2} k_{1}^{2}\right)\right) \\
& k_{2}^{z}=f_{2}\left(t_{n}+c_{z}^{2} \tau, I_{n}+\tau\left(a_{z 1}^{2} k_{1}^{2}+a_{z 2}^{2} k_{2}^{2}+a_{z 3}^{2} k_{3}^{2}+\ldots+a_{z, z-1}^{2} k_{z-1}^{2}\right),\right. \\
& k_{1}^{3}=f_{3}\left(t_{n}, I_{n}\right) \\
& k_{2}^{3}=f_{3}\left(t_{n}+c_{2}^{3} \tau, R_{n}+\tau\left(a_{21}^{3} k_{1}^{3}\right)\right) \\
& k_{3}^{z}=f_{3}\left(t_{n}+c_{z}^{3} \tau, R_{n}+\tau\left(a_{z 1}^{3} k_{1}^{3}+a_{z 2}^{3} k_{2}^{3}+a_{z 3}^{3} k_{3}^{3}+\ldots+a_{z, z-1}^{3} k_{z-1}^{3}\right),\right. \\
& k_{1}^{4}=f_{4}\left(t_{n}, E_{n}\right), \\
& k_{2}^{4}=f_{4}\left(t_{n}+c_{2}^{4} \tau, E_{n}+\tau\left(a_{21}^{4} k_{1}^{4}\right)\right), \\
& k_{3}^{z}=f_{3}\left(t_{n}+c_{z}^{4} \tau, E_{n}+\tau\left(a_{z 1}^{4} k_{1}^{4}+a_{z 2}^{4} k_{2}^{4}+a_{z 3}^{4} k_{4}^{4}+\ldots+a_{z, z-1}^{4} k_{z-1}^{4}\right),\right.
\end{aligned}
$$

where $\tau$ is the step of the time and $S\left(t_{n}\right), I\left(t_{n}\right), R\left(t_{n}\right)$ and $E\left(t_{n}\right)$ are the susceptible cases of pandemic individual, infectious cases of pandemic individual, recovered cases of pandemic individual and exposed cases of pandemic individual respectively at the moment $t_{n}$ and $S\left(t_{n+1}\right), I\left(t_{n+1}\right), R\left(t_{n+1}\right)$ and $E\left(t_{n+1}\right)$ are susceptible cases of pandemic individual, infectious cases of pandemic individual, recovered cases of pandemic individual and exposed cases of the pandemic individual respectively at the moment $t_{n+1}$. The coefficients $\operatorname{lmj}$ and cuj, appeared in the equation of the Runge-Kutta method (Equations (6)-(21)), are the weights and the nodes of the function expansion and $a_{q u}^{j}$ are the Runge-Kutta matrix coefficients, all the previous coefficients can be found using Butcher tableau and the weights are under the following condition $\sum_{m=1}^{m=z} l^{j} m=1$. In the flowchart presented in Figure 1, we illustrate the procedures followed for estimating the predictors of SARS-CoV-2 using the previous method. First, we write the collected data of the populations (susceptible, exposed, infectious and recovery) as matrices, then, we find the coefficient of the model and after that we find the SEIR predictors. Here, we note that we have to apply the conservation condition (Equation (5)) at each step of the calculations.

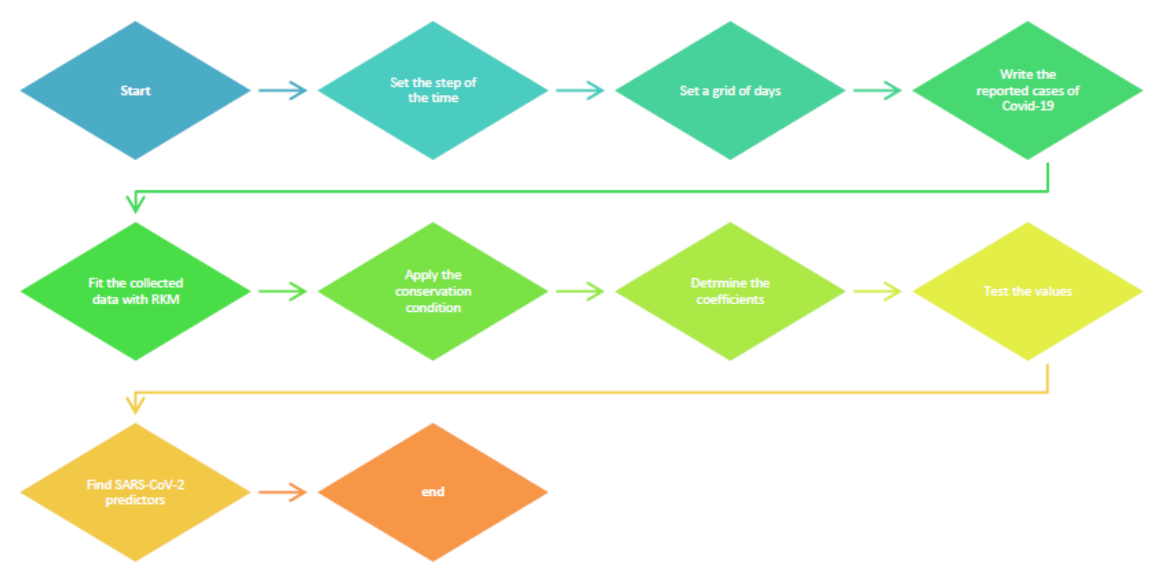

Figure 1. Schematic chart of the algorithm used in the study 


\section{Results and Discussion}

We calculated coefficient of exposing, coefficient of infection, coefficient of recovery and coefficient of mortality of the new coronavirus disease for the United States, which is located in north America, Russia which is located between Asia and Europe, the United Kingdom, which is located at north-west Europe, Brazil, which is located in south America, Spain, which is located at south-west Europe, Bahrain, which is located in Arabian Gulf, Egypt, which is located in Africa, Iran, which is located in west Asia, Cyprus, which is located in Mediterranean, India, which located in south Asia, France, which is located in west Europe and the Syrian Arab Republic, which is located at East of Mediterranean based on the reported data of the all cases of the new coronavirus disease in each country. After that we found the incubation periods of SARS-CoV-2. We illustrate the period of incubation values for the United States, Russia, the United Kingdom, Brazil, Spain, Bahrain, Egypt, Iran, Cyprus, India, France and the Syrian Arab Republic In Table 1, besides, the climate type of each country is illustrated in the same table. In addition to the incubation period values, we estimated the values of the steady state population of SARS-CoV-2 for the previous countries and we illustrated the results of this estimations in Table 2. Finally, the values of the critical immunisation threshold of SARS-CoV-2 were estimated for the previous countries and the results of this threshold were illustrated in Table 3 with the basic reproduction number values of SARS-CoV-2 for these countries.

Table 1. The values of the incubation period of SARS-CoV-2 in the United States, Russia, the United Kingdom, India, France, Brazil, Spain, Bahrain, Egypt, Iran, Cyprus and the Syrian Arab Republic and the geographical location of each country.

\begin{tabular}{c|c|c} 
The country & The location of the country & $P_{i} d$ \\
\hline The United States & North America & 4.643 \\
\hline India & South-Asia & 8.000 \\
\hline Russia & Easter Europe-Northern Asia & 4.000 \\
\hline The United Kingdom & North-West Europe & 6.406 \\
\hline France & West-Europe & 2.560 \\
\hline Brazil & South America & 7.143 \\
\hline Spain & South-Western Europe & 8.117 \\
\hline Bahrain & Arabian Gulf & 2.500 \\
\hline Egypt & North Africa & 10.00 \\
\hline Iran & West Asia & 5.000 \\
\hline Cyprus & Mediterranean & 10.00 \\
\hline The Syrian Arab Republic & East of Mediterranean & 10.00
\end{tabular}

As we see from Table 1, the largest value of the incubation periods of SARS-CoV-2 is 10.00 days which returns to some middle east countries and the smallest value is 2.500 days which returns to Bahrain. Besides, we find that the average value of the incubation period of SARS-CoV-2 is 6.531 days.

Table 2. 2 The values of the steady state population of SARS-CoV-2 in the United States, Russia, the United Kingdom, Brazil, Spain, Bahrain, Egypt, Iran, Cyprus and the Syrian Arab Republic and the climate type of each country.

\begin{tabular}{c|c|c} 
The country & The climate & $S_{M}$ \\
\hline The United States & Changeable & 0.310 \\
\hline Russia & Continental & 0.448 \\
\hline The United Kingdom & Temperate & 0.416 \\
\hline Brazil & Tropical & 0.366 \\
\hline Spain & Temperate & 0.666 \\
\hline Bahrain & Arid & 0.329 \\
\hline Egypt & Arid & 0.623 \\
\hline Iran & Arid & 0.497 \\
\hline Cyprus & Mediterranean & 0.636 \\
\hline The Syrian Arab Republic & Mediterranean & 0.450
\end{tabular}


We see from Table 2 that the values of the steady state population of the new corona virus disease are located between 0.310 for the united states and 0.666 for Spain and the average value of the steady state population equals to 0.474 for different location countries.

Table 3. The values of the critical immunization threshold and the basic reproduction number of SARS-CoV-2 in the United States, Russia, the United Kingdom, Brazil, Spain, Bahrain, Egypt, Iran, Cyprus and the Syrian Arab Republic.

\begin{tabular}{c|c|c} 
The country & $n_{\mathcal{C}}$ & $R_{o}$ \\
\hline The United States & 0.690 & 3.224 \\
\hline Russia & 0.552 & 2.231 \\
\hline The United Kingdom & 0.584 & 2.403 \\
\hline Brazil & 0.634 & 2.731 \\
\hline Spain & 0.334 & 1.501 \\
\hline Bahrain & 0.671 & 3.040 \\
\hline Egypt & 0.337 & 1.606 \\
\hline Iran & 0.503 & 2.012 \\
\hline Cyprus & 0.364 & 1.573 \\
\hline The Syrian Arab Republic & 0.550 & 2.222
\end{tabular}

As we see from Table 3, the value of the basic reproduction number of the new coronavirus pandemic for the United States and Bahrain are the greatest values in the previous different countries and the value of the basic reproduction number of the new coronavirus pandemic for Spain and Cyprus are the smallest between the previous different countries which returns to the high numbers of the infectious cases in the United States and the number of the cases with the new corona virus disease in Bahrain comparing to the number of people to the begging of 2021. Also, we see that the values of the reproduction numbers of the new coronavirus pandemic are in the range [1.5-3.5] for the pervious different countries with the climate and the geographic locations. Alternatively, we see from the same table that the critical immunization threshold are located between 0.334 which returns to Spain and 0.690 which returns to the United States. In addition, we see that the average values of the SARS-CoV-2 critical immunization threshold and the SARS-CoV-2 basic reproduction number are 0.526 and 2.254 respectively.

\section{Conclusions}

We employed one of the epidemiological models, namely, the SEIR model for estimating some important indicators values of the new coronavirus pandemic in twelve different countries, namely, the United States, Russia, the United Kingdom, Brazil, Spain, Bahrain, Egypt, Iran, Cyprus, India, France and the Syrian Arab Republic. The choice of countries was based on the different geographical location of countries on the earth and different with the type of the weather in each one. We used the recorded data of the collected cases of SARS-CoV-2 in the aforementioned countries, up to the beginning of 2021, to find the coefficient of exposing, the coefficient of infection, the coefficient of recovery and the coefficient of mortality of the new coronavirus pandemic for every country. First, we calculated the incubation period values of the new coronavirus pandemic in the aforementioned countries based on the coefficients of the SEIR model and the Rungee-Kutta simulation method. We found that the average value of the incubation period was about 6.531 days and the values of the period of infection (Table 1) were located in the interval [2.5-10] where the largest values return to Egypt, Cyprus and the Syrian Arab Republic and the smallest one returns to Bahrain. Also, we calculated the steady state population values of the new corona virus disease (Table 2) and found that the values of this population ratio are located in the interval [0.3-0.7] for the different location countries.

Finally, we found the critical immunization threshold values and the basic reproduction number values of the new coronavirus disease for ten of the previous countries. We found that the basic reproduction number values of the new coronavirus pandemic (Table 3) are in the interval [1.5-3.5] for the different countries. Besides, we found that the values of the basic reproduction number of the new coronavirus pandemic for the United States and Bahrain were the greatest values while the smallest values were for Spain and Cyprus. Alternatively, we found that the values of the critical immunization threshold are located in the interval [0.3-0.7] for those countries. We can use the same method for estimating the predictors of SARS-CoV-2 for other countries with different numbers of the new coronavirus pandemic, however, we chose the previous 
countries to clarify the relation between the incubation periods of SARS-CoV-2 with the geographical location and the climate of the country.

\section{Abbreviations}

- SARS : Severe acute respiratory syndrome.

- SARS - CoV - 2 : Severe acute respiratory syndrome-Corona Virus-2.

- SIRD : The susceptible-infected-recovered-dead.

- SEIR : The susceptible-exposed-infected-recovered.

- SVEIS : The susceptible-vaccinated-exposed-infected-susceptible.

- RK : Rungee-Kutta.

Conflicts of Interest: "The author declares no conflict of interest."

\section{References}

[1] Lounis, M., \& Bagal, D. K. (2020). Estimation of SIR model's parameters of COVID-19 in Algeria. Bulletin of the National Research Centre, 44(1), 1-6.

[2] Neto, O. P., Kennedy, D. M., Reis, J. C., Wang, Y., Brizzi, A. C. B., Zambrano, G. J., \& Zângaro, R. A. (2021). Mathematical model of COVID-19 intervention scenarios for São Paulo-Brazil. Nature Communications, 12(1), 1-13.

[3] Ebohon, O., Obienu, A. C., Irabor, F., Amadin, F. I., \& Omoregie, E. S. (2021). Evaluating the impact of COVID-19 pandemic lockdown on education in Nigeria: Insights from teachers and students on virtual/online learning. Bulletin of the National Research Centre, 45(1), 1-11.

[4] Ganiny, S., \& Nisar, O. (2021). Mathematical modeling and a month ahead forecast of the coronavirus disease 2019 (COVID-19) pandemic: An indian scenario. Modeling Earth Systems and Environment, 7(1), 29-40.

[5] Aidoo, E. N., Ampofo, R. T., Awashie, G. E., Appiah, S. K., \& Adebanji, A. O. (2021). Modelling COVID-19 incidence in the African sub-region using smooth transition autoregressive model. Modeling Earth Systems and Environment, 2021. https://doi.org/10.1007/s40808-021-01136-1.

[6] Gunawan, A. A. (2021). Forecasting Social Distancing impact on COVID-19 in Jakarta using SIRD Model. Procedia Computer Science, 179, 662-669.

[7] Al-Raeei, M. (2021). The basic reproduction number of the new coronavirus pandemic with mortality for India, the Syrian Arab Republic, the United States, Yemen, China, France, Nigeria and Russia with different rate of cases. Clinical Epidemiology and Global Health, 9, 147-149.

[8] Matadi, M. B. (2020). On the integrability of the sird epidemic model. Communications in Mathematical Biology and Neuroscience, 2020, Article ID 5869275, 10 pages, https:/ / doi.org/10.1155/2020/5869275.

[9] Lounis, M., \& Al-Raeei M. (2021). Estimation of epidemiological indicators of COVID-19 in Algeria with an SIRD model. Eurasian Journal of Medicine and Oncology, 5(1), 54-58.

[10] Al-Raeei, M. (2020). The forecasting of COVID-19 with mortality using SIRD epidemic model for the United States, Russia, China, and the Syrian Arab Republic. AIP Advances, 10(6), 065325, https://doi.org/10.1063/5.0014275.

[11] Youssef, H. M., Alghamdi, N. A., Ezzat, M. A., El-Bary, A. A., \& Shawky, A. M. (2020). A modified SEIR model applied to the data of COVID-19 spread in Saudi Arabia. AIP Advances, 10(12), 125210, https://doi.org/10.1063/5.0029698.

[12] Ghostine, R., Gharamti, M., Hassrouny, S., \& Hoteit, I. (2021). An extended seir model with vaccination for forecasting the covid-19 pandemic in saudi arabia using an ensemble kalman filter. Mathematics, 9(6), 636, https://doi.org/10.3390/math9060636.

[13] Kounchev, O., Simeonov, G., \& Kuncheva, Z. (2021). Scenarios for the spread of COVID-19 analyzed by the TVBG-SEIR spline model. BIOMATH, 10(1), 2103087, https:/ / doi.org/10.11145/j.biomath.2020.03.087.

[14] Adedire, O. \& Ndam, J. N. (2021). A model of dual latency compartments for the transmission dynamics of COVID-19 in Oyo state, Nigeria. Engineering and Applied Science Letters, 4(1), 1-13.

[15] Kermack, W. O. (1927). A Contirbution to the Mathematical Theory of Epedimic. Procedding of the royal socity of London $115,700-721$.

[16] Das, T., Srivastava, P. K., \& Kumar, A. (2021). Nonlinear dynamical behavior of an SEIR mathematical model: Effect of information and saturated treatment. Chaos: An Interdisciplinary Journal of Nonlinear Science, 31(4), 043104, https:/ /doi.org/10.1063/5.0039048.

[17] Abdelaziz, M. A., Ismail, A. I., Abdullah, F. A., \& Mohd, M. H. (2020). Codimension one and two bifurcations of a discrete-time fractional-order SEIR measles epidemic model with constant vaccination. Chaos, Solitons E Fractals, 140, 110104, https:/ /doi.org/10.1016/j.chaos.2020.110104. 
[18] Li, Q., Du, Y., Li, Z., Hu, J., Hu, R., Lv, B., \& Jia, P. (2021). HK-SEIR model of public opinion evolution based on communication factors. Engineering Applications of Artificial Intelligence, 100, 104192, https://doi.org/10.1016/j.engappai.2021.104192.

[19] Alonso-Quesada, S., De la Sen, M., \& Ibeas, A. (2012, February). A Vaccination Strategy Based on a State Feedback Control Law for Linearizing SEIR Epidemic Models. In International Joint Conference on Biomedical Engineering Systems and Technologies (pp. 195-209). Springer, Berlin, Heidelberg.

[20] Zhao, C., Li, M., Wang, J., \& Ma, S. (2021). The mechanism of credit risk contagion among internet P2P lending platforms based on a SEIR model with time-lag. Research in International Business and Finance, 57, 101407, https://doi.org/10.1016/j.ribaf.2021.101407.

[21] Kumar, S., Kumar, R., Osman, M. S., \& Samet, B. (2021). A wavelet based numerical scheme for fractional order SEIR epidemic of measles by using genocchi polynomials. Numerical Methods for Partial Differential Equations, 37(2), 1250-1268.

[22] Alabkari, A., Kourrad, A., Adnaoui, K., Laaroussi, A. E. A., Soukher, N., \& Bennar, A. (2021). Control of a reaction-diffusion system: Application on a seir epidemic mode. Journal of Mathematical and Computational Science, 11(2), 1601-1628.

[23] Wang, L., Liu, Z., Guo, C., Li, Y., \& Zhang, X. (2021). New global dynamical results and application of several SVEIS epidemic models with temporary immunity. Applied Mathematics and Computation, 390, 125648, https:/ / doi.org/10.1016/j.amc.2020.125648.

[24] Al-Raeei, M. (2018). Using methods of statistical mechanics in the study of soft condensed matter materials and complex structures. Damascus, Syrian Arab Republic: Damascus University Publishing.

[25] Al-Raeei, M. (2021). Numerical simulation of the force of infection and the typical times of SARS-CoV-2 disease for different location countries. Modeling Earth Systems and Environment, 2021, https://doi.org/10.1007/s40808-020-01075-3. 